Conclusion We have shown that hands can be vehicles for transmission of Streptococcus pneumoniae and that wet particles increased transmission. This reinforces the imperative for good hand hygiene especially in populations at risk of invasive pneumococcal disease or pneumonia such as young children, elderly and immunosuppressed people

\section{P120 WHICH SCORING SYSTEM IS BETTER AT PREDICTING LIKELY MORTALITY AND INTENSIVE CARE UNIT (ICU) ADMISSION IN COMMUNITY ACQUIRED PNEUMONIA RELATED SEPSIS?}

F Groutsi-Allwright, G Chellappah, S Niroshan, S Abburu, A Asour, A Choudhury. Barking, Havering and Redbridge University Hospitals NHS Trust, Romford, UK

\subsection{6/thoraxjnl-2017-210983.262}

Introduction and Aim Sepsis and pneumonia commonly present together, however the preferred clinical scoring system to use is unclear. NEWS (National Early Warning Score) and SIRS (Systemic Inflammatory Response Syndrome) and SOFA (Sequential Organ Failure Assessment) scores can be used to evaluate patients admitted with sepsis. Due to complexity of SOFA scoring, qSOFA has been proposed for routine practice. ${ }^{1}$ We compared sepsis scores in patients with community acquired pneumonia related sepsis (CAP sepsis) and compared this to sepsis from other causes (non-CAP sepsis). We also evaluated how sepsis scoring systems compared with the CURB-65 score to predict mortality and ICU admission in CAP sepsis.

Methods Medical records were audited between 01/09/201620/03/2017 at Barking Havering and Redbridge University Hospitals NHS Trust. Adult patients were included if ICD-10 codes on discharge/death were Sepsis A40/A41. Physiological and blood parameters were collected at time of trigger from medical/electronic records. All plain chest x-rays (CXR) and CT-chest scans were reviewed independently by two respiratory registrars to confirm consolidation. We calculated the positive predictive value (PPV) and negative predictive value (NPV) of each sepsis scoring system against two outcome measures; i)ICU Admission <24 hours ii)mortality(death) at 30 days.

Outcomes/Results We identified 114 cases of sepsis. Median age-78 years. Male:female ratio 56:58. We found 22/114 (19\%) of patients had a diagnosis of CAP sepsis with CXR/ CT-chest confirming consolidation $<48$ hours from admission. Scores for CAP sepsis $(n=22)$ triggered more often than nonCAP $\operatorname{sepsis}(n=92)$ for NEWS $\geq 4 \quad(77 \%$ vs $54 \%, p=0.05)$,
SOFA $\geq 2(86 \%$ vs $54 \%, p<0.01)$. For CAP sepsis and nonCAP sepsis, the PPV and NPV for each sepsis score including CURB-65 were calculated for $<24$ hour ICU admission and 30 day mortality (Table.1). In patients with CAP sepsis, scores for NEWS/SIRS/SOFA had a high PPV for ICU admission and mortality. CURB-65 $>3$ had a low PPV but higher NPV.

Conclusion Patients with CAP sepsis triggered more often with NEWS $\geq 4$ and SOFA $\geq 2$ compared to non-CAP sepsis. Our data suggests that NEWS $\geq 4$, SIRS $\geq 2$,SOFA $\geq 2$ or CURB$65 \geq 2$ may be used as initial screening for CAP sepsis. However, all had low NPV for ICU admission and 30 day mortality. Further studies are needed to evaluate the best scoring system to assess clinical severity for CAP sepsis and avoid unnecessary duplication.

\section{REFERENCE}

1. Singer $\mathrm{M}$ et al. Third International Consensus Definitions for Sepsis and Septic Shock: JAMA 2016:315(8):801-810.

\section{P121 THE RESPIRATORY INFECTIONS TEAM - A NOVEL PARADIGM IN THE MANAGEMENT OF COMMUNITY- ACQUIRED PNEUMONIA}

G Cresswell, T Bewick. Derby Teaching Hospitals NHS Foundation Trust, Derby, UK

\subsection{6/thoraxjnl-2017-210983.263}

Background The recent British Thoracic Society national audit of community-acquired pneumonia (CAP) has shown that guideline concordance and antimicrobial stewardship remain poor in the UK. Furthermore, mis- and over-diagnosis are common. ${ }^{1}$

Methods A Respiratory Infections Team was developed, comprising a specialist nurse supported by a respiratory consultant and antimicrobial pharmacist. Its aims were to review patients admitted to hospital with CAP, and to a) identify patients with low severity CAP for remote outpatient management with early supported discharge; b) facilitate streamlined antibiotic regimens using bedside point-of-care (POC) tests (BinaxNOW pneumococcal and legionella urinary antigens, and nasopharyngeal swab for influenza PCR), reducing total amount of antibiotic prescribed both in route and spectrum; and c) improve diagnostic accuracy, communication with clinical coders, and concordance with the BTS pneumonia care bundle. Outcomes were compared with a prospective cohort of consecutive radiographically-confirmed CAP admissions from winter 2013/2014.

Abstract P120 Table 1 Triggering of sepsis scoring systems and CURB-65 scores for predicting ICU admission within 24 hours and 30 day mortality in patients with community acquired pneumonia related sepsis and non-community acquired pneumonia related sepsis

\begin{tabular}{|c|c|c|c|c|c|c|c|c|c|c|}
\hline \multirow[t]{3}{*}{ Sepsis Scoring System } & \multicolumn{5}{|l|}{ CAP sepsis $(n=22)$} & \multicolumn{5}{|l|}{ non-CAP sepsis ( $n=92)$} \\
\hline & \multirow[t]{2}{*}{ Total cases triggered $\mathrm{n}(\%)$} & \multicolumn{2}{|c|}{ Admission to ICU $<24$ hours } & \multicolumn{2}{|c|}{ Mortality<30 days } & \multirow[t]{2}{*}{ Total cases triggered n (\%) } & \multicolumn{2}{|c|}{ Admission to ICU $<24$ hours } & \multicolumn{2}{|c|}{ Mortality $<30$ days } \\
\hline & & PPV & NPV & PPV & NPV & & PPV & NPV & PPV & NPV \\
\hline NEWS $\geq 4$ & $17(77.2 \%)$ & $100.0 \%$ & $33.3 \%$ & $91.6 \%$ & $40.0 \%$ & $50(54.3 \%)$ & $57.1 \%$ & $45.8 \%$ & $65.3 \%$ & $49.5 \%$ \\
\hline $\operatorname{SIRS} \geq 2$ & $18(81.8 \%)$ & $100.0 \%$ & $26.6 \%$ & $91.6 \%$ & $30.0 \%$ & $66(71.7 \%)$ & $100.0 \%$ & $30.5 \%$ & $80.7 \%$ & $30.7 \%$ \\
\hline SOFA $\geq 2$ & $19(86.3 \%)$ & $100.0 \%$ & $20.0 \%$ & $100.0 \%$ & $30.0 \%$ & $50(54.3 \%)$ & $85.7 \%$ & $48.2 \%$ & $76.9 \%$ & $55.4 \%$ \\
\hline$q S O F A \geq 2$ & $7(31.8 \%)$ & $57.1 \%$ & $80.0 \%$ & $41.6 \%$ & $80.0 \%$ & $15(16.3 \%)$ & $28.5 \%$ & $84.7 \%$ & $34.6 \%$ & $90.7 \%$ \\
\hline CURB $65 \geq 2$ & $16(72.7 \%)$ & $85.7 \%$ & $33.3 \%$ & $83.3 \%$ & $40.0 \%$ & \multicolumn{5}{|c|}{ CURB 65 was not used for non-CAP sepsis } \\
\hline CURB $65 \geq 3$ & $7(31.8 \%)$ & $28.5 \%$ & $66.6 \%$ & $41.6 \%$ & $80.0 \%$ & & & & & \\
\hline
\end{tabular}

